\title{
Rate Coefficient Determination in Charge Transfer Reactions
}

\author{
Marie-Christine Bacchus-Montabonel ${ }^{*}$, Ezinvi Baloïtcha ${ }^{\ddagger}$, Michèle Desouter-Lecomte ${ }^{\frac{t^{*}}{4} \text { and }}$ \\ Nathalie Vaeck ${ }^{\dagger}$
}

* Laboratoire de Spectrométrie Ionique et Moléculaire, UMR 5579, CNRS et Université Lyon I, 43 Bd. du 11 Novembre 1918, 69622 Villeurbanne Cedex, France. bacchus@lasim.univ-lyon1.fr \$ Laboratoire de Chimie Théorique, Université de Paris Sud (Orsay), France and Département de Chimie, Université de Liège, Institut de Chimie B6, Sart-Tilman, B-4000, Liège 1, Belgium. $\dagger$ Laboratoire de Chimie Physique Moléculaire, CP 160/09 Université Libre de Bruxelles, 50 Av. F. Roosevelt, B-1050 Bruxelles, Belgium.

Received: 18 December 2001/ Accepted: 17 January 2002/ Published: 28 March 2002

\begin{abstract}
The development of experimental ion-trap techniques provides the opportunity to compare directly theoretical rate coefficients to experimental data in the low-temperature regime. In the present work, we consider the ion-atom collisional systems, $\mathrm{N}^{2+}, \mathrm{O}^{2+}, \mathrm{Si}^{3+}$ and $\mathrm{Si}^{4+}$ on a He target, in order to examine the present status of the agreement between theory and experiment.
\end{abstract}

Keywords: ion-atom collisions; wave packet method; rate coefficients.

\section{Introduction}

Charge transfer processes involving low-energy multiply charged ions in collision with atomic or molecular targets are determinant in laboratory and astrophysical plasmas. In such processes, an electron is generally captured in an excited state of the ion, followed by line emission. Line intensities provide important information on the electron temperature, density and spatial distributions in the emitting region of the plasma. In some cases, however, the electron may be captured directly in a ground state of the ion, whereupon ionization may occur rapidly via the inverse charge transfer process [1,2]. A precise understanding of charge transfer processes is thus essential for the determination of the ion abundances and ionization balance of the plasmas.

From a theoretical point of view, the description of very low-velocity processes requires a complete quantum mechanical treatment of the dynamics of both electrons and nuclei. The first approach extensively used is the resolution of the stationary close-coupling equations [3]. We have analyzed recently 
the efficiency of a time-dependent wave packet method [4-6] which provides a clear and physical insight into the dynamics of the processes and may be particularly interesting for polyatomic systems since it allows the possibility of developing a fully quantal mechanical treatment for some degrees of freedom, the other ones being treated classically. In this method, a wave packet initially located in the entrance channel is propagated on the coupled electronic potential energy curves; the collision matrix elements are then extracted by Hankel transform of the time signal obtained from a wave packet stored in the asymptotic region. The main features of this approach are summarised in section II.

The development of modern facilities such as crossed- or merged-beam techniques [7,8], and more recently ion-trap experiments [9] lead to possible experimental investigations in the very low collision energy range. Experiments using laser-induced-plasma ion sources and ion storage [9] provide for the first time a direct determination of rate coefficients at low temperatures, of the order of $10^{3}$ to $10^{4} \mathrm{~K}$. Since ions are produced by laser ablation from a solid target, no source gas is required; this avoids the potential source of systematic error due to reactions between the ions and the source gas. The ions are then stored in a cylindrical radiofrequency ion trap and the mass selectivity of the ion trap allows for the storage of a specific ion group with no other concomitant ions present. Laser ablation produces the ions in a variety of excited states, but they rapidly cascade to their ground state and low-lying metastable states through allowed transitions and collisional deexcitations with plasma electrons.

Such experimental techniques provide the possibility to compare directly theoretically determined rate coefficients at low-temperature to experimental data. The $\mathrm{O}^{2+} / \mathrm{O}^{3+}[9-11], \mathrm{N}^{2+}[12,13]$, and $\mathrm{Si}^{3+} / \mathrm{Si}^{4+}[14,15]$ ions colliding with gaseous targets of $\mathrm{He}, \mathrm{H}_{2}, \mathrm{~N}_{2}$, and $\mathrm{CO}$ and, more recently, $\mathrm{He}^{+}$ [16] and $\mathrm{H}_{3}{ }^{+}$[17] ions colliding with molecular targets of $\mathrm{N}_{2}, \mathrm{CO}$ and $\mathrm{CH}_{4}$ have been investigated experimentally. From the theoretical point of view, ion/atom collisions involving $\mathrm{O}^{2+}, \mathrm{N}^{2+}, \mathrm{Si}^{3+}$ and $\mathrm{Si}^{4+}$ on He have been extensively studied. The comparison with the experimental results appears to be quite different from one ion to another, showing good agreement in some cases, but very large discrepancies in others. The purpose of this review is to investigate the collisional systems, $\mathrm{O}^{2+}+\mathrm{He}[3,18-20], \mathrm{N}^{2+}$ $+\mathrm{He}$ [20-21], $\mathrm{Si}^{3+}+\mathrm{He}[1,2,23,24,28]$ and $\mathrm{Si}^{4+}+\mathrm{He}$ [5,6,23,24-29] in order to exhibit the difficulties encountered both in the theoretical and experimental approaches. The systems $\mathrm{O}^{2+} / \mathrm{He}, \mathrm{N}^{2+} / \mathrm{He}, \mathrm{Si}^{3+}$ $/ \mathrm{He}$ are treated by the close coupling formalism. The wave packet method is applied to the $\mathrm{Si}^{4+} / \mathrm{He}$ case.

\section{Wave packet dynamics}

The convention $\hbar=1$ is adopted throughout. The theoretical model used to solve the timedependent Schrödinger equation

$$
i \partial[R \psi(\vec{r}, \vec{R}, t)] / \partial t=\hat{H}[R \psi(\vec{r}, \vec{R}, t)],
$$

where $\hat{H}$ is the total transformed Hamiltonian $\left(\hat{H}=R H R^{-1}\right)$ which has been described in detail by Vaeck et al. [4]. In summary, the wave function corresponding to the entry channel $i$ is given by the superposition :

$$
\psi_{i}^{K}(\vec{r}, \vec{R}, t)=1 / R \sum_{\alpha \Lambda} \chi_{\alpha \Lambda}^{K}(R, t) \phi_{\alpha \Lambda}(\vec{r} ; R) Y_{\Lambda}^{K}(\theta, \phi),
$$

where $K$ is the total angular momentum with $\Lambda$ its projection on the internuclear axis. The nuclear wave functions are the product of an angular part, $Y^{K}{ }_{\Lambda}$, and a radial part which contains the entire time dependence, $\chi_{\alpha \Lambda}^{K}$. The electronic wavefunctions $\phi_{\alpha \Lambda}$ can be expressed either in the adiabatic $\left(\phi_{\alpha \Lambda}=a_{\alpha \Lambda}\right)$ 
or in the diabatic $\left(\phi_{\alpha \Lambda}=d_{\alpha \Lambda}\right)$ representation. The unitary matrix $\mathbf{F}$ transforming the adiabatic representation in the diabatic one is obtained by solving the equation :

$$
\frac{\partial}{\partial R} \mathbf{F}+\mathbf{P F}=0
$$

where the matrix $\mathbf{P}$ contains the radial coupling matrix elements.

The corresponding time-dependent functions, $\chi_{\alpha \Lambda}^{K}(R, t)=A_{\alpha \Lambda}^{K}(R, t)$ or $\chi_{\alpha \Lambda}^{K}(R, t)=D_{\alpha \Lambda}^{K}(R, t)$ are solutions of the coupled equations :

$$
i \frac{\partial \chi_{\alpha \Lambda}^{K}(R, t)}{\partial t}=\sum_{\beta \Lambda^{\prime}} \mathbf{H}_{\alpha \Lambda, \beta \Lambda^{\prime}}^{K} \chi_{\beta \Lambda^{\prime}}^{K}(R, t) .
$$

When Coriolis coupling is neglected, the $\mathbf{H}^{K}$ matrix is written, respectively

$$
\mathbf{H}^{K}=T_{R} \mathbf{I}+\mathbf{E}^{a}+\frac{K(K+1)-2 \Lambda^{2}}{2 \mu R^{2}} \mathbf{I}-\frac{1}{2 \mu}\left[\mathbf{Q}+2 \mathbf{P} \frac{\partial}{\partial R}\right]
$$

in the adiabatic representation or

$$
\mathbf{H}^{K}=T_{R} \mathbf{I}+\mathbf{H}^{d}+\frac{K(K+1)-2 \Lambda^{2}}{2 \mu R^{2}} \mathbf{I}
$$

in the diabatic one. $T_{R}$ is the nuclear kinetic operator, $T_{R}=-1 / 2 \mu \partial^{2} / \partial \mathrm{R}^{2} . \mathbf{E}^{a}$ is the diagonal matrix of the adiabatic energies, and $\mathbf{H}^{d}$ is the matrix of the electronic Hamiltonian in the diabatic basis set. The radial coupling $\mathbf{P}$ matrix elements, $P_{\alpha \beta}=<a_{\alpha} / \partial / \partial R / a_{\beta}>$ have been calculated using a numerical differentiation method. The double derivative matrix elements $Q_{\alpha \beta}$ have been computed from the following relationship [30,31] :

$$
Q_{\alpha \beta}=\left\langle a_{\alpha}\left|\frac{\partial^{2}}{\partial R^{2}}\right| a_{\beta}\right\rangle=\frac{\partial}{\partial R}\left\langle a_{\alpha}\left|\frac{\partial}{\partial R}\right| a_{\beta}\right\rangle+\sum_{k}\left\langle a_{\alpha}\left|\frac{\partial}{\partial R}\right| a_{k}\right\rangle\left\langle a_{k}\left|\frac{\partial}{\partial R}\right| a_{\beta}\right\rangle,
$$

The radial wavefunction for the initial state $(\alpha=i)$ at time $t=0$ is given by a Gaussian wave packet centered initially in $R_{0}$ with a width $\sigma_{R}$ at half maximum, and a mean relative kinetic energy $\varepsilon_{0}$ corresponding to the wave number $k_{0}=\left(2 \mu \varepsilon_{0}\right)^{1 / 2}$ :

$$
\chi_{\alpha=i}(R, t=0)=1 /\left(\pi \sigma_{R}^{2}\right)^{1 / 4} \exp \left(-i k_{0} R\right) \exp \left(-1 / 2\left[R-R_{0} / \sigma_{R}\right]^{2}\right) .
$$

In any method based on wave packets, the extraction of energy resolved functions requires the computation of the amplitude of the initial wave packet on the stationary states

$$
\eta_{\alpha}(\mathrm{E})=\left\langle\Psi_{\alpha \mathrm{E}}^{+} \mid \chi_{\alpha 0}\right\rangle
$$

$\chi_{\alpha 0}$ is located in the asymptotic region so that only its limit is used

$$
\Psi_{\alpha \mathrm{E}}^{\mathrm{K}}(\mathrm{R}, \mathrm{r}) \underset{\mathrm{R} \rightarrow \infty}{\longrightarrow} \sqrt{\frac{\mu}{2 \pi \mathrm{k}_{\alpha}}}\left[\mathrm{h}_{\mathrm{K}}^{-}\left(\mathrm{k}_{\alpha} \mathrm{R}\right) \phi_{\alpha}^{\mathrm{K}}-\sum_{\beta} \mathrm{S}_{\beta \alpha}^{\mathrm{K}}(\mathrm{E}) \mathrm{h}_{\mathrm{K}}^{+}\left(\mathrm{k}_{\alpha} \mathrm{R}\right) \phi_{\alpha}^{\mathrm{K}}\right]
$$

where $h^{ \pm}\left(k_{\alpha} R\right)$ are the Hankel-Riccatti outgoing/incoming wave functions corresponding to the long range potential $\mathrm{K}(\mathrm{K}+1) / 2 \mu \mathrm{R}^{2}$ and $\mathrm{k}_{\alpha}=\sqrt{2 \mu\left(\mathrm{E}-\mathrm{E}_{\alpha}\right)}$. When $\chi_{\alpha 0}$ does not contain any outgoing momentum, one has

$$
\eta_{\alpha}(E)=\sqrt{\frac{\mu}{2 \pi k_{\alpha}}} \int_{0}^{\infty} h_{K}\left(k_{\alpha} R\right) g_{\alpha}(R) d R
$$

where $g_{\alpha}(R)$ is a Gaussian function in the entrance channel. 
Different strategies are possible in order to obtain the collision matrix elements. They have been compared in [6]

(i) The spectral projection method. The $S_{\beta \alpha}^{K}(E)$ elements are extracted from the functions

$$
\psi_{\alpha \mathrm{E}}^{+}(\mathrm{R}, \mathrm{r})=\frac{\mathrm{i}}{2 \pi} \mathrm{G}^{+}(\mathrm{E}) \chi_{\alpha 0}(\mathrm{R}, \mathrm{r})
$$

where $\mathrm{G}^{+}(\mathrm{E})=(\mathrm{E}-\hat{\mathrm{H}}+\mathrm{i} 0)^{-1}$ is the resolvant. The general relation is

$$
S_{\beta \alpha}^{K}(E)=\frac{i}{2 \pi} \frac{\left\langle\chi_{\beta}\left|G^{+}(E)\right| \chi_{\alpha 0}\right\rangle}{\eta_{\beta}^{*}(E) \eta_{\alpha}(E)}
$$

When the probe nuclear wave packet in the asymptotical region $\chi_{\beta}$ is chosen to be the delta function $\mathrm{g}_{\beta}(\mathrm{R})=\delta\left(\mathrm{R}-\mathrm{R}_{\infty}\right)$, one gets

$$
\mathrm{S}_{\beta \alpha}^{\mathrm{K}}(\mathrm{E})=-\sqrt{\frac{2 \pi \mathrm{k}_{\beta}}{\mu}} \mathrm{h}_{\mathrm{K}}^{-}\left(\mathrm{k}_{\beta} \mathrm{R}_{\infty}\right) \Phi_{\beta \alpha}\left(\mathrm{R}_{\infty}, \mathrm{E}\right) / \eta_{\alpha}(\mathrm{E})
$$

where

$$
\Phi_{\beta \alpha}(\mathrm{R}, \mathrm{E})=\left\langle\phi_{\beta}^{\mathrm{K}} \mid \psi_{\alpha \mathrm{E}}^{+}\right\rangle_{\mathrm{r}, \Omega}
$$

The resolvant in (1) can be comptuted by time independent or time dependent methods. In the first case, $\mathrm{G}^{+}(\mathrm{E})$ is expanded on the Chebyshev polynomials by using the Mandelshtam and Taylor modified recursion [32]

$$
\mathrm{G}^{+}(\mathrm{E}) \chi_{\alpha 0}=\sum_{\mathrm{n}=0}^{\infty} \mathrm{a}_{\mathrm{n}}(\mathrm{E}) \mathrm{T}_{\mathrm{n}}\left(\hat{\mathrm{H}}_{\text {scale }}\right) \chi_{\alpha 0}
$$

where $\hat{\mathrm{H}}_{\text {scale }}$ is the scaled Hamiltonian mapping the spectrum in the range [-1, 1] [33]. In the second case, $\mathrm{G}^{+}(\mathrm{E})$ is expressed as the Fourier transform of the evolution operator

$$
\mathrm{G}^{+}(\mathrm{E}) \chi_{\alpha 0}=-\mathrm{i} \int_{0}^{\infty} \exp (-\mathrm{iH} \mathrm{H}) \chi_{\alpha 0} \exp (\mathrm{iEt}) \mathrm{dt}
$$

The wave packets are then propagated on the coupled electronic potentials using the split-operator technique [34] in the diabatic representation and the more cpu time consuming Chebyshev scheme in the adiabatic basis set. The difference comes from the structure of the $\mathbf{H}^{K}$ matrix in the two representations. The Chebyshev method only requires the computation of the $\hat{H} \Psi$ and is thus able to account for any kind of differential operators.

(ii) The flux method. Within this approach, the square modulus of the $S_{\beta \alpha}^{K}$ (E) elements are related to the average value of the flux operator evaluated in the asymptotical region at $\mathrm{R}=\mathrm{R}_{\mathrm{c}}$

$$
\left\langle\Psi_{\alpha \mathrm{E}}^{+}\left|\hat{\mathrm{P}}_{\beta}^{\mathrm{K}} \hat{\mathrm{F}} \hat{\mathrm{P}}_{\beta}^{\mathrm{K}}\right| \Psi_{\alpha \mathrm{E}}^{+}\right\rangle=\frac{1}{2 \pi}\left|\mathrm{S}_{\beta \alpha}^{\mathrm{K}}(\mathrm{E})\right|^{2}
$$

where $\hat{\mathrm{P}}_{\beta}^{\mathrm{K}}$ is the projector on the electronic state and

$$
\hat{\mathrm{F}}=\frac{-\mathrm{i}}{2 \mu}\left(\frac{\partial}{\partial \mathrm{R}} \delta\left(\mathrm{R}-\mathrm{R}_{\mathrm{c}}\right)+\delta\left(\mathrm{R}-\mathrm{R}_{\mathrm{c}}\right) \frac{\partial}{\partial \mathrm{R}}\right)
$$

By adding a complex absorbing potential $\hat{\mathrm{H}} \rightarrow \hat{\mathrm{H}}-\mathrm{i} \hat{\mathrm{W}}$ in the asymptotical range, Jäckle and Meyer have derived the following expression [35]

$$
\left|S_{\beta \alpha}^{K}(E)\right|^{2}=\frac{2}{\pi \eta_{\alpha}(E)^{2}} \Re e \int_{0}^{\infty} f_{\beta}(\tau) \exp (-i E \tau) d \tau
$$

where

$$
\mathrm{f}_{\beta}(\tau)=\int_{0}^{\infty}\left\langle\chi_{\alpha}(\tau+\mathrm{t})\left|\hat{\mathrm{P}}_{\beta}^{\mathrm{K}} \hat{\mathrm{W}} \hat{\mathrm{P}}_{\beta}^{\mathrm{K}}\right| \chi_{\alpha}(\mathrm{t})\right\rangle \mathrm{dt}
$$


After extracting the $S_{\beta \alpha}^{K}(E)$ elements, the final expression of the state selective charge exchange cross section is given by :

$$
\sigma_{\beta, i}(E)=\frac{\pi}{\left(2 L_{i}+1\right) k_{i}^{2}(E)} \sum_{K} \sum_{\Lambda \Lambda^{\prime}}(2 K+1) \times \mid i\left(S_{\beta \Lambda^{\prime}, i \Lambda}^{K}(E)-\delta_{\beta, i} \delta_{\Lambda, \Lambda^{\prime}}\right)^{2},
$$

where $\left(2 L_{i}+1\right)$ is the degeneracy of the initial state.

The rate coefficients, $k(T)$ are then calculated by averaging the cross sections over a Maxwellian velocity distribution at temperature $T$.

$$
k(T)=\left(\frac{2}{k_{B} T}\right)^{3 / 2}\left(\frac{1}{\pi \mu}\right)^{1 / 2} \int_{0}^{E} E \sigma(E) \exp \left(-E / k_{B} T\right) d E
$$

\section{The $\mathrm{N}^{2+} / \mathrm{He}$ system}

The experimental study of the electron-capture from the ground state of helium atoms by $\mathrm{N}^{2+}(2 \mathrm{p})$ ${ }^{2} \mathrm{P}^{\mathrm{o}}$ ions has received recently a lot of attention [36-38]. Four reaction channels and two entry channels are involved in the transfer mechanism :

$$
\begin{aligned}
{ }^{2} \Sigma^{+},{ }^{2} \Pi \quad \mathrm{N}^{2+}(2 \mathrm{p}){ }^{2} \mathrm{P}^{\mathrm{o}}+\mathrm{He}\left(1 \mathrm{~s}^{2}\right){ }^{1} \mathrm{~S} & \rightarrow \mathrm{N}^{+}\left(2 \mathrm{p}^{2}\right){ }^{3} \mathrm{P}+\mathrm{He}^{+}(1 \mathrm{~s}) & { }^{2} \Pi \\
& \rightarrow \mathrm{N}^{+}\left(2 \mathrm{p}^{2}\right){ }^{1} \mathrm{D}+\operatorname{He}^{+}(1 \mathrm{~s}) & { }^{2} \Sigma^{+},{ }^{2} \Pi \\
& \rightarrow \mathrm{N}^{+}\left(2 \mathrm{p}^{2}\right){ }^{1} \mathrm{~S}+\operatorname{He}^{+}(1 \mathrm{~s}) & { }^{2} \Sigma^{+}
\end{aligned}
$$

The potential energy curves have been determined by Lafyatis et al. [22] using state-averaged multiconfiguration self-consistent-field (MCSCF) calculations with Slater-type function basis sets.
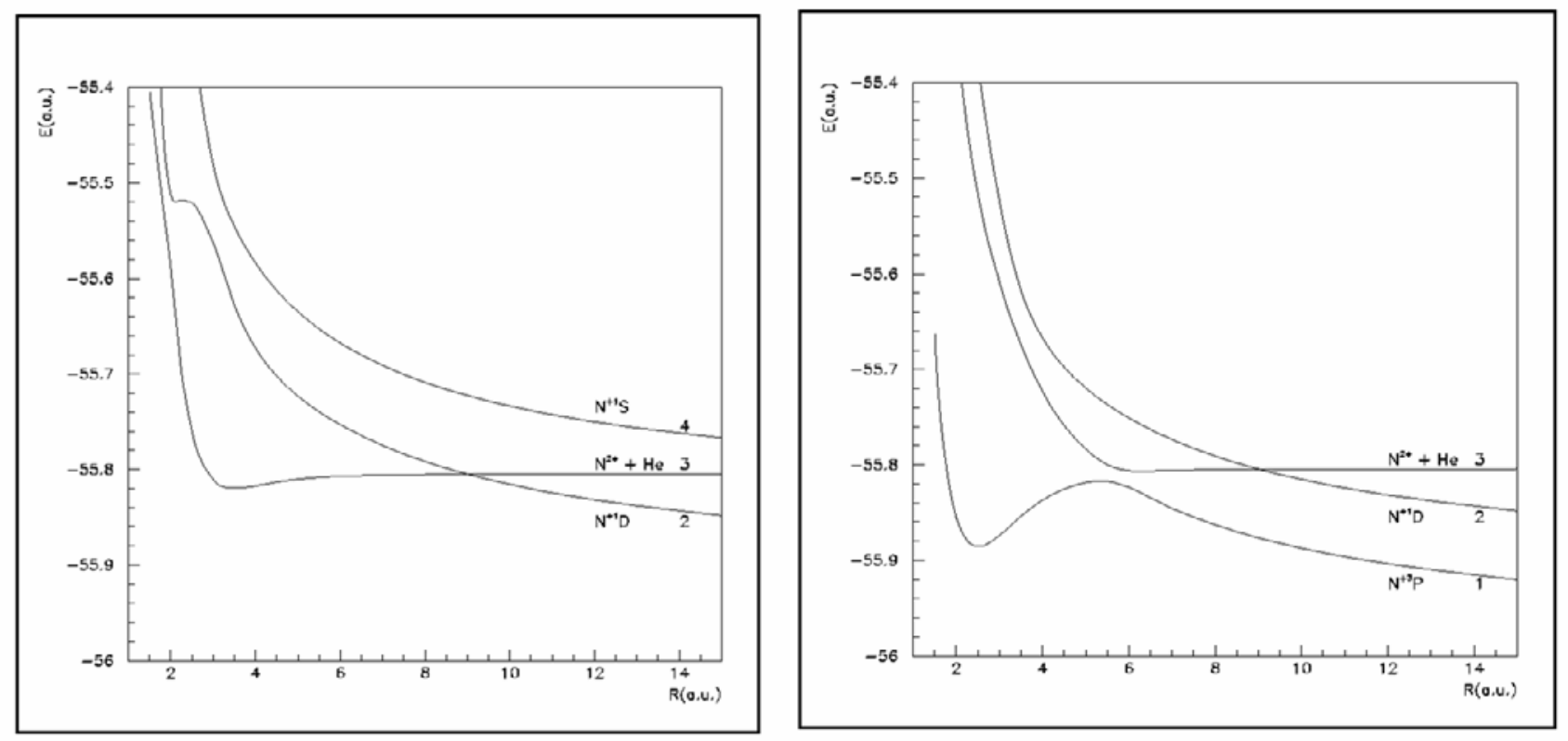

Fig. 1a,b : Adiabatic potential energy curves for respectively the ${ }^{2} \Sigma^{+}$and ${ }^{2} \Pi$ states of $\mathrm{N}^{2+}+\mathrm{He}$ [22]. $1:{ }^{2} \Pi$ state dissociating in $\mathrm{N}^{+}\left(2 \mathrm{p}^{2}\right){ }^{3} \mathrm{P}+\mathrm{He}^{+}(1 \mathrm{~s}) ; 2:{ }^{2} \Sigma$ and ${ }^{2} \Pi$ states dissociating in $\mathrm{N}^{+}\left(2 \mathrm{p}^{2}\right){ }^{1} \mathrm{D}+$ $\mathrm{He}^{+}(1 \mathrm{~s}) ; 3$ : entry channel $\mathrm{N}^{2+}(2 \mathrm{p}){ }^{2} \mathrm{P}^{\mathrm{o}}+\mathrm{He} ; 4:{ }^{2} \Sigma$ state dissociating in $\mathrm{N}^{+}\left(2 \mathrm{p}^{2}\right){ }^{1} \mathrm{~S}+\mathrm{He}^{+}(1 \mathrm{~s})$. 
The results displayed in Fig. 1a,b show clearly a very sharp avoided crossing in the ${ }^{2} \Sigma$ and ${ }^{2} \Pi$ symmetry between the entry channel and the $\mathrm{N}^{+}\left(2 \mathrm{p}^{2}\right){ }^{1} \mathrm{D}+\mathrm{He}^{+}(1 \mathrm{~s})$ level around $\mathrm{R}=9$ a.u. A smoother one is also observed in the ${ }^{2} \Pi$ manifold with the $\mathrm{N}^{+}\left(2 \mathrm{p}^{2}\right){ }^{3} \mathrm{P}+\mathrm{He}^{+}(1 \mathrm{~s})$ channel around $\mathrm{R}=6$ a.u. The ${ }^{2} \Sigma^{+}$, $\mathrm{N}^{+}\left(2 \mathrm{p}^{2}\right){ }^{1} \mathrm{~S}+\mathrm{He}^{+}(1 \mathrm{~s})$ level crosses the entry channel at very large interatomic distances, around 30 a.u. Therefore its influence on the charge transfer process is negligible.

The charge transfer cross sections have been evaluated using the close-coupling formalism [21] and the rate coefficient may be compared to the recent multichannel Landau-Zener calculations of Wang et al. [20] and to the recent ion-trap experimental result of Fang and Kwong [12]. These results are presented in Fig. 2. They show clearly that the two exit channels $\mathrm{N}^{+}\left(2 \mathrm{p}^{2}\right){ }^{1} \mathrm{D}$ and $\mathrm{N}^{+}\left(2 \mathrm{p}^{2}\right){ }^{3} \mathrm{P}$ provide important contributions to the total rate coefficient, $\mathrm{N}^{+}\left(2 \mathrm{p}^{2}\right){ }^{3} \mathrm{P}$ being the dominant channel at higher temperatures. The experimental temperature of the ions $T_{i}$ determined from the mean energy of the ions in

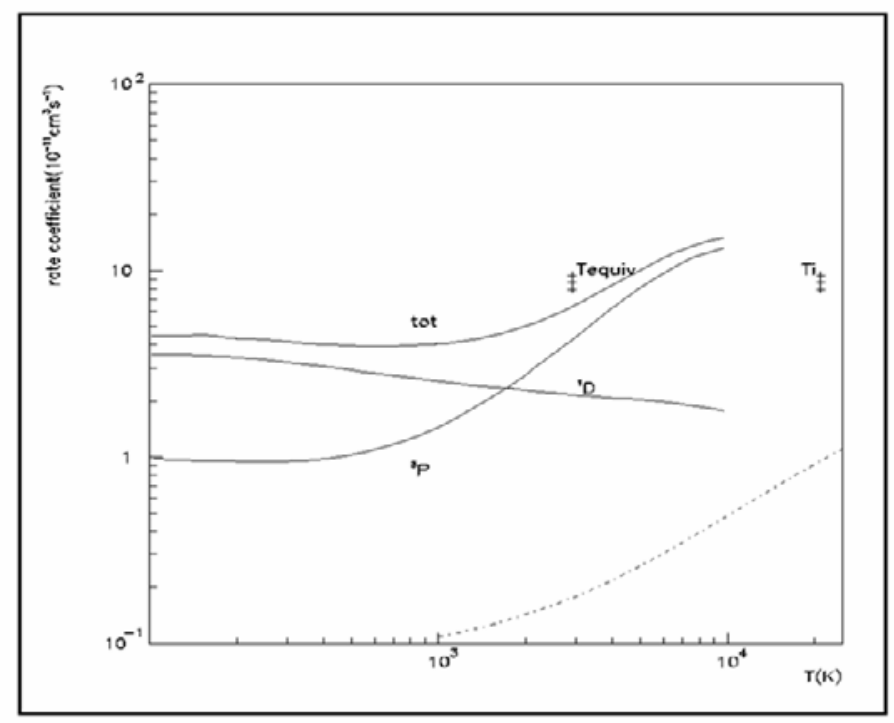

Fig. 2 : Rate coefficients as a function of temperature for $\mathrm{N}^{2+}+$ He. Comparison of close-coupling calculations [21] (full curves) and multichannel Landau-Zener approach [20] (dotted-broken curve) with ion-trap experiment at $\mathrm{T}_{\mathrm{i}}$ and $\mathrm{T}_{\text {equiv }}[12]$.

the trap may be quite different in the presence of neutral atoms of helium produced at room temperature. Fang and Kwong proposed to introduce an equivalent temperature $\mathrm{T}_{\text {equiv }}$ obtained from the mean relative velocity between the ion at temperature $T_{i}$ and the neutral atom at temperature $T_{n}$ [12]. Both temperatures have been displayed on Fig. 2, and differ by about a power of ten, $2900 \mathrm{~K}$ for $\mathrm{T}_{\text {equiv }}$ and $2.110^{4} \mathrm{~K}$ for $\mathrm{T}_{\mathrm{i}}$. The close-coupling result [21] appears to be in good agreement with the ion-trap experimental data of Fang and Kwong [12] when the $T_{\text {equiv }}$ is considered. On the other hand, the multichannel Landau-Zener approach [20] underestimates by more than an order of magnitude the closecoupling calculation.

\section{The $\mathrm{O}^{2+} / \mathrm{He}$ system}

The capture of one electron by the ground state $\mathrm{O}^{2+}{ }^{3} \mathrm{P}$ ion from He also involves four reaction channels, corresponding to the ${ }^{3} \Pi$ and ${ }^{3} \Sigma^{-}$molecular symmetry of the entry channels: 


$$
\begin{aligned}
{ }^{3} \Sigma^{-}, \Pi \quad \mathrm{O}^{2+}\left(2 \mathrm{p}^{2}\right){ }^{3} \mathrm{P}+\mathrm{He}\left(1 \mathrm{~s}^{2}\right){ }^{1} \mathrm{~S} & \rightarrow \mathrm{O}^{+}\left(2 \mathrm{p}^{3}\right){ }^{2} \mathrm{P}^{\mathrm{o}}+\mathrm{He}^{+}(1 \mathrm{~s}){ }^{3} \Pi \text {; peak B } \\
& \rightarrow \mathrm{O}^{+}\left(2 \mathrm{p}^{3}\right){ }^{2} \mathrm{D}^{\mathrm{o}}+\mathrm{He}^{+}(1 \mathrm{~s}){ }^{3} \Sigma^{-},{ }^{3} \Pi \text {; peak C } \\
& \rightarrow \mathrm{O}^{+}\left(2 \mathrm{p}^{3}\right){ }^{4} \mathrm{~S}^{\mathrm{o}}+\mathrm{He}^{+}(1 \mathrm{~s}){ }^{3} \Sigma^{-} \text {; peak D }
\end{aligned}
$$

where the labels B, C, D correspond to the peaks in the translational energy spectrum of McLaughlin et al. [39]. The potential energy curves determined by ab-initio calculations using the CIPSI algorithm with Gaussian basis sets [40] are displayed in Fig. 3a,b. Similar results have been obtained by Kimura et al. [18] with MRD-CI (multireference single- and double-excitation configuration interaction) calculations with a large basis set. The potential energy curves show a sharp avoided crossing around 5.5 a.u. between the ${ }^{3} \Pi, \mathrm{O}^{+}\left(2 \mathrm{p}^{3}\right){ }^{2} \mathrm{P}^{\mathrm{o}}+\mathrm{He}^{+}(1 \mathrm{~s})$ level and the ${ }^{3} \Pi$ entry channel, and a smoother one at about 4.5 a.u. between the ${ }^{3} \Pi, \mathrm{O}^{+}\left(2 \mathrm{p}^{3}\right){ }^{2} \mathrm{P}^{\mathrm{o}}+\mathrm{He}^{+}(1 \mathrm{~s})$ and $\mathrm{O}^{+}\left(2 \mathrm{p}^{3}\right)^{2} \mathrm{D}^{\mathrm{o}}+\mathrm{He}^{+}(1 \mathrm{~s})$ exit channels. An avoided crossing between the ${ }^{3} \Sigma^{-}, \mathrm{O}^{+}\left(2 \mathrm{p}^{3}\right){ }^{2} \mathrm{D}^{\mathrm{o}}+\mathrm{He}^{+}(1 \mathrm{~s})$ level and the ${ }^{3} \Sigma^{-}$entry channel is also observed at about 4.5 a.u.
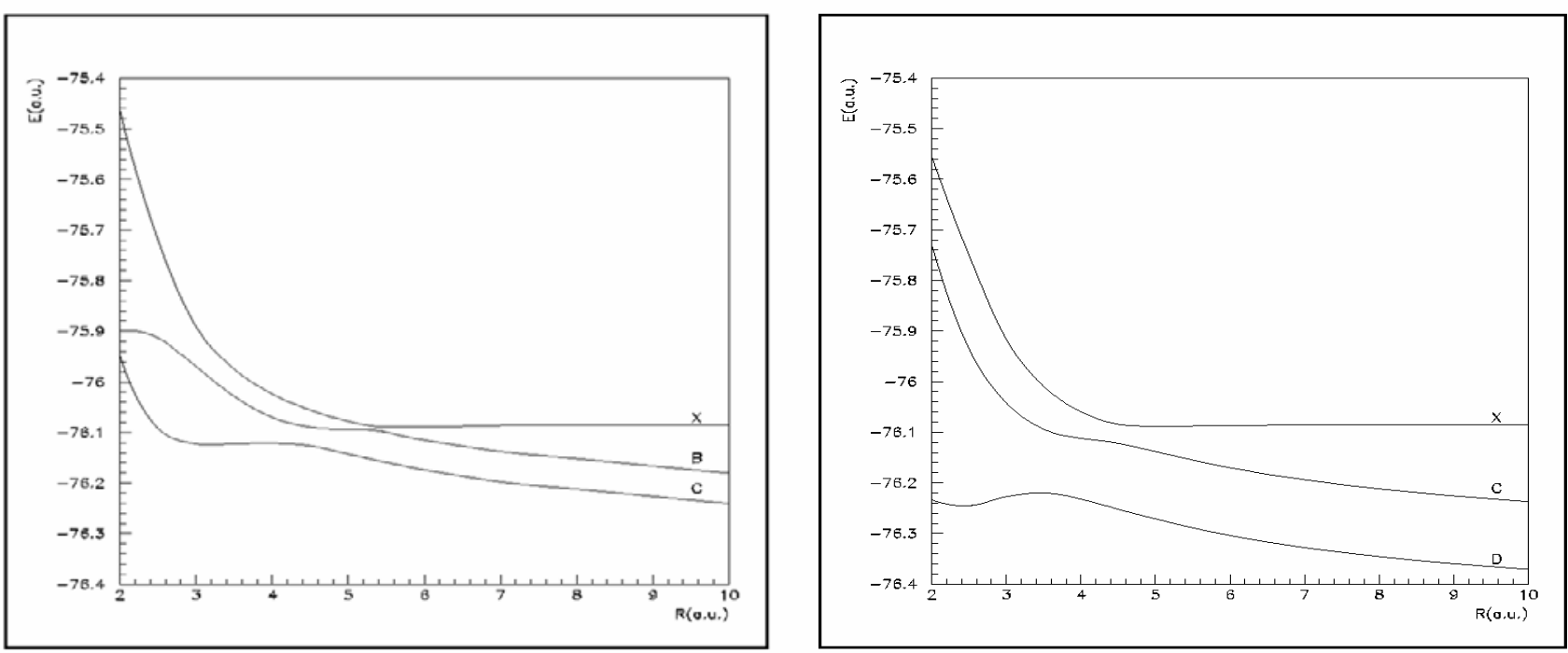

Fig. 3a,b : Adiabatic potential energy curves for respectively the ${ }^{3} \Sigma^{-}$and ${ }^{3} \Pi$ states of $\mathrm{O}^{2+}+\mathrm{He}[40]$. (labels defined in the text)

A quantum mechanical close-coupling calculation [3] of the cross sections and the rate coefficients may be compared to a similar calculation of Kimura et al. [18] and to the experimental data of Kwong et al. $[9,10]$. These data can also be compared to the previous calculation of Butler et al. [19] and the recent multichannel Landau-Zener calculations of Wang et al [20]. The results are displayed in Fig. 4. As far as the close-coupling calculations are concerned, the results of Gargaud et al [3] are in quite good agreement with the extensive calculation of Kimura et al. [18] for the $\mathrm{O}^{2+}{ }^{3} \mathrm{P}$ ground state ion. Nevertheless, these results appear to provide rate coefficients largely overestimated with regard to the previous calculation of Butler et al. [19] and the multichannel Landau-Zener approach [20], similarly to the case of the $\mathrm{N}^{2+}+\mathrm{He}$ collisional system. The close coupling results appear to be two orders of magnitude higher than the ion-trap experiment of Fang and Kwong [10], considering either $\mathrm{T}_{\mathrm{i}}$ (2.0 $\left.10^{4} \mathrm{~K}\right)$ or $\mathrm{T}_{\text {equiv }}(4200 \mathrm{~K})$. These results obtained from very accurate molecular calculations are about a factor two larger than the previous experimental value performed using drift tube techniques [41] which lies one order of magnitude above the results of Fang and Kwong. However, the rate coefficients calculated by Kimura et al. [18] for the $\mathrm{O}^{2+}\left(2 \mathrm{p}^{2}\right){ }^{1} \mathrm{D}$ excited entry channel appear to be of the 


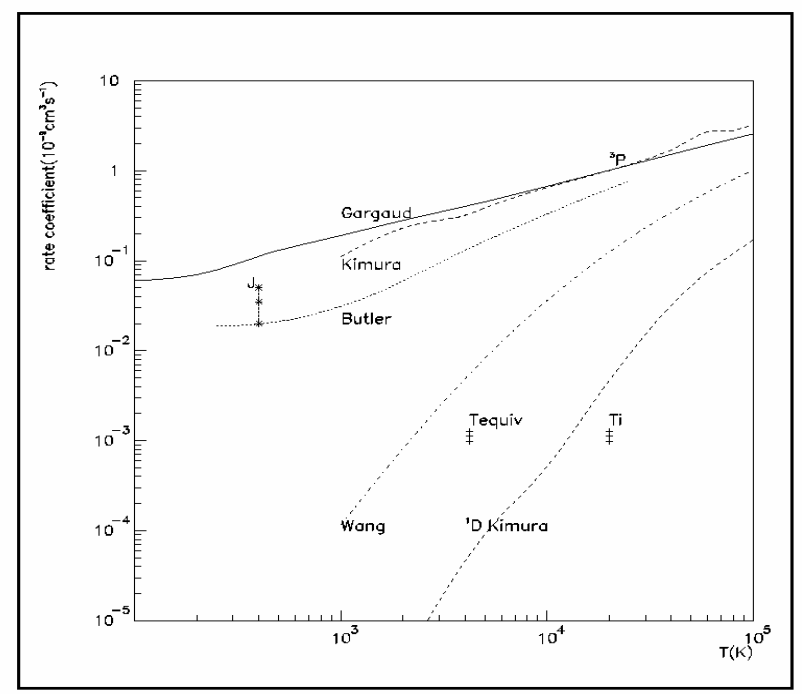

Fig. 4 : Rate coefficients as a function of temperature for $\mathrm{O}^{2+}+$ He. Comparison of close-coupling calculations, Gargaud et al. [3] (full curve) ; Kimura et al. [18] (broken curves) ; Butler et al. [19] (dotted curve) and multichannel Landau-Zener approach, Wang et al. [20] (dotted-broken curve) with ion trap experiment at $\mathrm{T}_{\mathrm{i}}$ and $\mathrm{T}_{\text {equiv }}[9,10]$ and drift tube technique experiments (J) [41].

same order of magnitude than the ion-trap result. A fraction of metastable ions in the beam could be a possible source of discrepancy between theoretical approaches and experimental data. Effectively, in the ion-trap experiment, ions produced by laser ablation can be in a variety of excited electronic states immediately after their production, but as measurements are performed $0.4 \mathrm{~s}$ after the ions have been produced and trapped, all the stored ions are expected to be in their ground-state [10] and the presence of excited states seems unlikely.

\section{The $\mathrm{Si}^{3+} / \mathrm{He}$ system}

The charge transfer recombinations of $\mathrm{Si}^{3+}$ ions with atomic hydrogen and helium are critical processes in determining the ionization balance of astrophysical plasmas as they lead to the formation of the ground-state ion and therefore can induce rapid ionization via the inverse process. The theoretical treatment of the $\mathrm{Si}^{3+}+\mathrm{He}$ collisional process involves the complete network of exit channels interacting with both the ground-state $\mathrm{Si}^{3+}(3 \mathrm{~s})$ and the excited $\mathrm{Si}^{3+}(3 \mathrm{p})+$ He entry channels. The total manifold of following channels must be considered :

\begin{tabular}{|c|c|c|c|}
\hline \multirow[t]{3}{*}{${ }^{2} \Sigma^{+}$} & $\mathrm{Si}^{3+}(3 \mathrm{~s}){ }^{2} \mathrm{~S}+\mathrm{He}\left(1 \mathrm{~s}^{2}\right){ }^{1} \mathrm{~S}$ & $\rightarrow \mathrm{Si}^{2+}\left(3 \mathrm{~s}^{2}\right){ }^{1} \mathrm{~S}+\mathrm{He}^{+}(1 \mathrm{~s}){ }^{2} \mathrm{~S}$ & ${ }^{2} \Sigma^{+}$ \\
\hline & & $\rightarrow \mathrm{Si}^{2+}(3 \mathrm{~s} 3 \mathrm{p}){ }^{3} \mathrm{P}^{\mathrm{o}}+\mathrm{He}^{+}(1 \mathrm{~s}){ }^{2} \mathrm{~S}$ & ${ }^{2} \Sigma^{+},{ }^{2} \mathrm{I}$ \\
\hline & & $\rightarrow \mathrm{Si}^{2+}(3 \mathrm{~s} 3 \mathrm{p}){ }^{1} \mathrm{P}^{\mathrm{o}}+\mathrm{He}^{+}(1 \mathrm{~s}){ }^{2} \mathrm{~S}$ & ${ }^{2} \Sigma^{+},{ }^{2} \Pi$ \\
\hline \multirow[t]{3}{*}{${ }^{2} \Sigma^{+},{ }^{2} \Pi$} & $\mathrm{Si}^{3+}(3 p){ }^{2} \mathrm{P}^{\mathrm{o}}+\mathrm{He}\left(1 \mathrm{~s}^{2}\right){ }^{1} \mathrm{~S}$ & $\rightarrow \mathrm{Si}^{2+}\left(3 \mathrm{~s}^{2}\right){ }^{1} \mathrm{~S}+\mathrm{He}^{+}(1 \mathrm{~s}){ }^{2} \mathrm{~S}$ & ${ }^{2} \Sigma^{+}$ \\
\hline & & $\rightarrow \mathrm{Si}^{2+}(3 \mathrm{~s} 3 \mathrm{p}){ }^{3} \mathrm{P}^{\mathrm{o}}+\mathrm{He}^{+}(1 \mathrm{~s})^{2} \mathrm{~S}$ & \\
\hline & & $\rightarrow \mathrm{Si}^{2+}(3 \mathrm{~s} 3 \mathrm{p}){ }^{1} \mathrm{P}^{\mathrm{o}}+\mathrm{He}^{+}(1 \mathrm{~s})^{2} \mathrm{~S}$ & ${ }^{2} \Sigma^{+},{ }^{2} \Pi$ \\
\hline
\end{tabular}

The potential energy curves have been calculated [1] by means of ab-initio configuration interaction methods based on the CIPSI algorithm with Gaussian basis sets constructed from McLean and Chan- 
dler [42]. A non-local pseudo-potential [43] has been used to represent the core electrons of the Si atom. The results are displayed in Fig. 5a,b. From a dynamical point of view, the ground-state entry channel ${ }^{2} \Sigma^{+}, \mathrm{Si}^{3+}(3 \mathrm{~s}){ }^{2} \mathrm{~S}+\mathrm{He}\left(1 \mathrm{~s}^{2}\right){ }^{1} \mathrm{~S}$ leads to a simple electron capture process with a pronounced avoided crossing with the ${ }^{2} \Sigma^{+}, \mathrm{Si}^{2+}\left(3 \mathrm{~s}^{2}\right){ }^{1} \mathrm{~S}+\mathrm{He}^{+}(1 \mathrm{~s}){ }^{2} \mathrm{~S}$ around $\mathrm{R}=6$ a.u.. It interacts also with the ${ }^{2} \Sigma^{+}$,
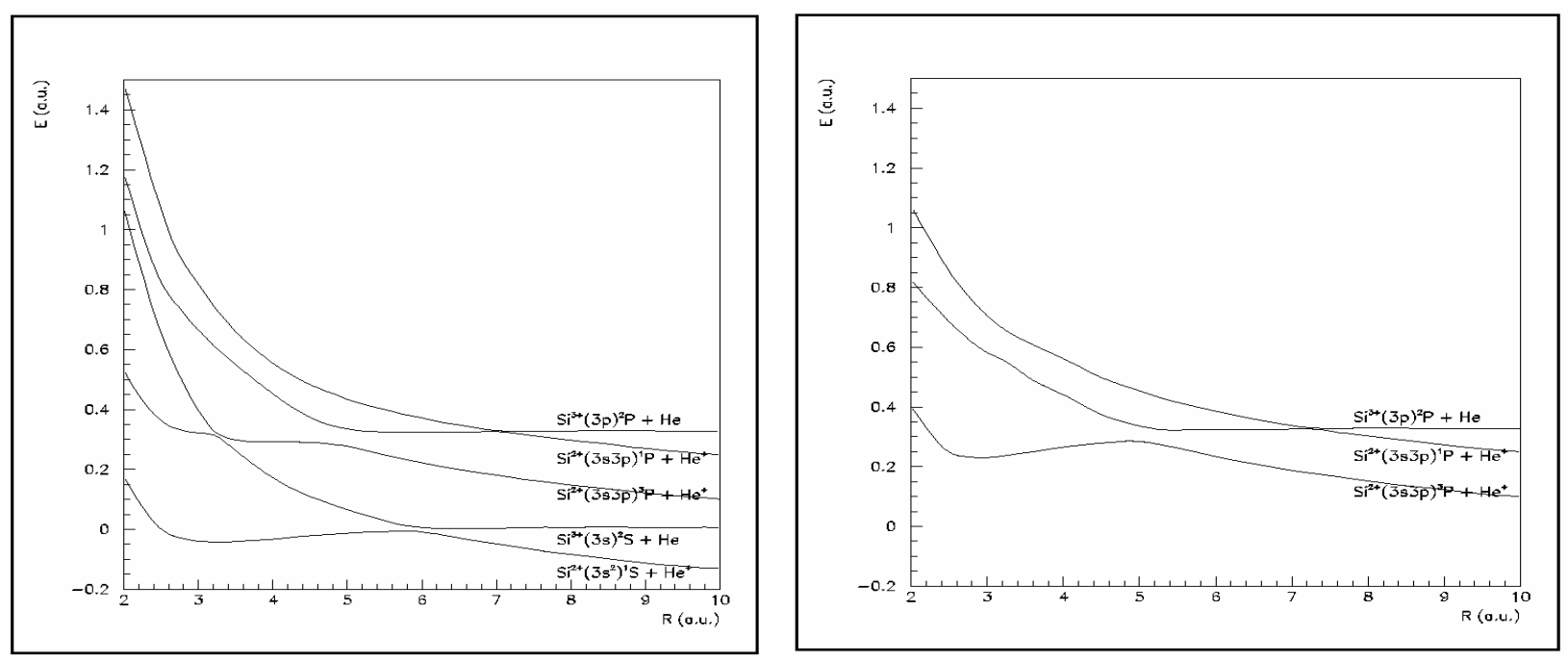

Fig. 5a,b : Adiabatic potential energy curves for respectively the ${ }^{2} \Sigma^{+}$and ${ }^{2} \Pi$ states of $\mathrm{Si}^{3+}+\mathrm{He}[1]$.

$\mathrm{Si}^{2+}(3 \mathrm{~s} 3 \mathrm{p}){ }^{3} \mathrm{P}^{\mathrm{o}}+\mathrm{He}^{+}(1 \mathrm{~s}){ }^{2} \mathrm{~S}$ level around $\mathrm{R}=3.2$ a.u. The excited $\mathrm{Si}^{3+}(3 \mathrm{p})$ entry channel shows important avoided crossings around $\mathrm{R}=7$ a.u. and 5 a.u. with respectively the $\mathrm{Si}^{2+}(3 \mathrm{~s} 3 \mathrm{p}){ }^{1} \mathrm{P}^{\mathrm{o}}$ and $\mathrm{Si}^{2+}(3 \mathrm{~s} 3 \mathrm{p})$ ${ }^{3} \mathrm{P}^{\mathrm{o}}$ channels, both in the ${ }^{2} \Sigma^{+}$and ${ }^{2} \Pi$ symmetry. These potential energy curves are in satisfactory agreement with the spin-coupled valence bond calculations of Clarke and Cooper [44].

From these molecular data, a quantum mechanical collisional treatment taking account simultaneously of all the levels involved in the process has been performed [1] and the rate coefficients are displayed in Fig. 6. They may be compared to the quantum mechanical close-coupling treatment of Stancil et al. [2] and to the previous Landau-Zener calculations of Butler and Dalgarno [24], as well as the experimental data of Fang and Kwong [14]. For the capture process by the ground-state $\mathrm{Si}^{3+}(3 \mathrm{~s})$ ion, a global agreement is observed between the Landau Zener calculations and the ab-initio treatments, showing a similar shape with a decrease of the rate coefficients with decreasing temperature. These results appear to be $35 \%$ to $47 \%$ lower than the ion-trap experimental data at $\mathrm{T}_{\text {equiv }}=3900 \mathrm{~K}$. Nevertheless, as already pointed out in the previous section, the determination of this equivalent temperature based on the assumption of a thermodynamic equilibrium between the ion at temperature $T_{i}=2.910^{4}$ $\mathrm{K}$ and a neutral atom at room temperature may be questioned. As $\mathrm{T}_{\mathrm{i}}$ is about two orders of magnitude larger than the room temperature of the helium gas, it should be rather close to the real temperature of the process. Both $\mathrm{T}_{\mathrm{i}}$ and $\mathrm{T}_{\text {equiv }}$ temperatures have been drawn on the graph and show clearly that an uncertainty on the temperature of the reactants in the trap can modify the conclusion on the agreement between theory and experiment. Furthermore, it is important to ask if the measurement of Fang and Kwong obtained using an ion trap with cylindrical symmetry can be compared with a calculation based on the use of a Maxwell-Boltzmann distribution. On the other hand, the calculated rate coefficients [1] from the excited $\mathrm{Si}^{3+}(3 \mathrm{p})$ entry channel appear to be of the same order of magnitude than experimental data points for both $\mathrm{T}_{\mathrm{i}}$ and $\mathrm{T}_{\text {equiv. }}$. This could suggest, as already pointed out for the $\mathrm{O}^{2+}$ ions, that the 


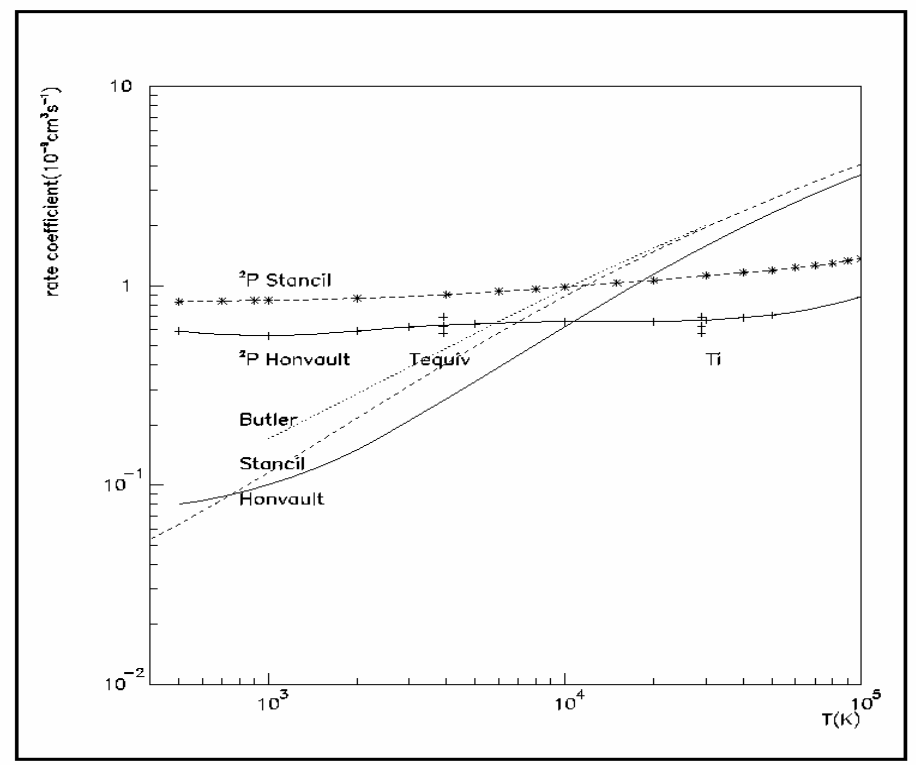

Fig. 6 : Rate coefficients as a function of temperature for $\mathrm{Si}^{3+}+\mathrm{He}$. Comparison of close coupling calculations, Honvault et al. [1] (full curves) ; Stancil et al. [2] (broken curves) and Landau-Zener approach, Butler et al. [24] (dotted curve) with ion trap experiment at $\mathrm{T}_{\mathrm{i}}$ and $\mathrm{T}_{\text {equiv }}$ [14].

presence of a fraction of excited ions in the trap could be a possible source of discrepancy between theoretical approaches and experimental results. Nevertheless, the life-time of $\mathrm{Si}^{3+}$ ions being about $1.3 \mathrm{~ns}$, one would expect all of the $3 \mathrm{p}$ states to have radiatively decayed to the ground state as ion-trap measurements are performed $0.4 \mathrm{~s}$ after the production of the ions. Measurements at different temperatures could however be particularly interesting, since the rate coefficients for the ground state $\mathrm{Si}^{3+}(3 \mathrm{~s})$ ion exhibit a very different temperature dependence than the $\mathrm{Si}^{3+}(3 \mathrm{p})$ entry channel which is almost constant with temperature.

\section{The $\mathrm{Si}^{4+} / \mathrm{He}$ system}

From a theoretical point of view, the most extensively studied system with regard to the ion-trap experiment of Kwong et al. [15], is undoubtedly the $\mathrm{Si}^{4+}+$ He collisional system [5,6,23,25-28]. This system presents a relatively simple molecular structure with three singlet electron capture channels :

$$
\begin{aligned}
& { }^{1} \Sigma^{+} \quad \mathrm{Si}^{4+}\left(2 \mathrm{p}^{6}\right){ }^{1} \mathrm{~S}+\mathrm{He}\left(1 \mathrm{~s}^{2}\right){ }^{1} \mathrm{~S} \rightarrow \quad \mathrm{Si}^{3+}(3 \mathrm{~s}){ }^{2} \mathrm{~S}+\mathrm{He}^{+}(1 \mathrm{~s}){ }^{2} \mathrm{~S} \quad{ }^{1} \Sigma^{+} \\
& \mathrm{Si}^{3+}(3 \mathrm{p}){ }^{2} \mathrm{P}^{\mathrm{o}}+\mathrm{He}^{+}(1 \mathrm{~s}){ }^{2} \mathrm{~S} \quad{ }^{1} \Sigma^{+},{ }^{1} \Pi
\end{aligned}
$$

The potential energy curves and couplings have been determined with the code MOLPRO [45] using a state-average complete active space self-consistent field (CASSCF) calculation followed by a multireference configuration interaction calculation (MRCI) with Gaussian basis set augmented with extra $\mathrm{f}$ orbitals [46]. A pseudopotential [47] has been used to describe the core orbitals of the Si atom. The corresponding potential energy curves are displayed in Fig 7. A sharp avoided crossing is clearly observed between the entry channel and the $\mathrm{Si}^{3+}(3 \mathrm{p}){ }^{2} \mathrm{P}^{\mathrm{o}}+\mathrm{He}^{+}(1 \mathrm{~s}){ }^{2} \mathrm{~S}$ level at about $\mathrm{R}=7$ a.u. and a smoother one around $\mathrm{R}=4.5 \mathrm{a}$.u. between the two $\mathrm{Si}^{3+}$ channels. These results are in good agreement 


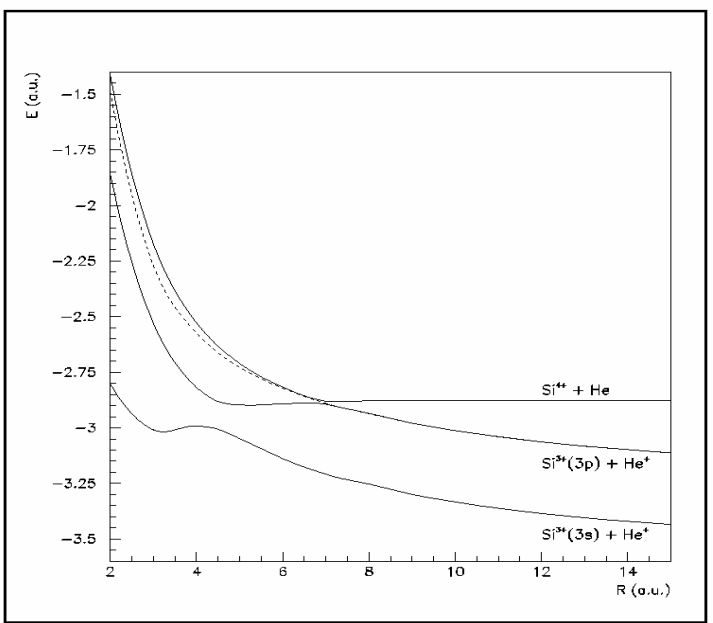

Fig. 7 : Adiabatic potential energy curves for the ${ }^{1} \Sigma^{+}$(full lines) and ${ }^{1} \Pi$ (dotted line) of $\mathrm{Si}^{4+}+\mathrm{He}$

with previous configuration interaction calculations using the CIPSI algorithm [25] and other ab-initio calculations, using the spin-coupled valence bond method (SCVB) $[26,44]$ or the configuration interaction MRD-CI method [27].

Wave packet propagation approaches [5,6] as summarized in part II has been applied in order to determine the cross sections and rate coefficients at low temperatures. The results are presented in Fig. 8 and compared to the time-independent close-coupling approaches [26,27,29]. The agreement between the theoretical calculations is good and presents a similar temperature dependence. The introduction of the Hankel-Ricatti functions [6] to replace the approximate plane wave functions [5] in extracting the S-matrix in the time-dependent approach improves the accuracy of the calculation. However, all the calculations appear to be in complete disagreement, by a two order of magnitude difference, with the experimental point of Fang and Kwong [15]. This is all the more surprising because recent cross section measurements at ion energies of 100-500 eV [28] appear to be in good agreement with theoretical calculations. Of course the problem of temperature determination still exists as shown

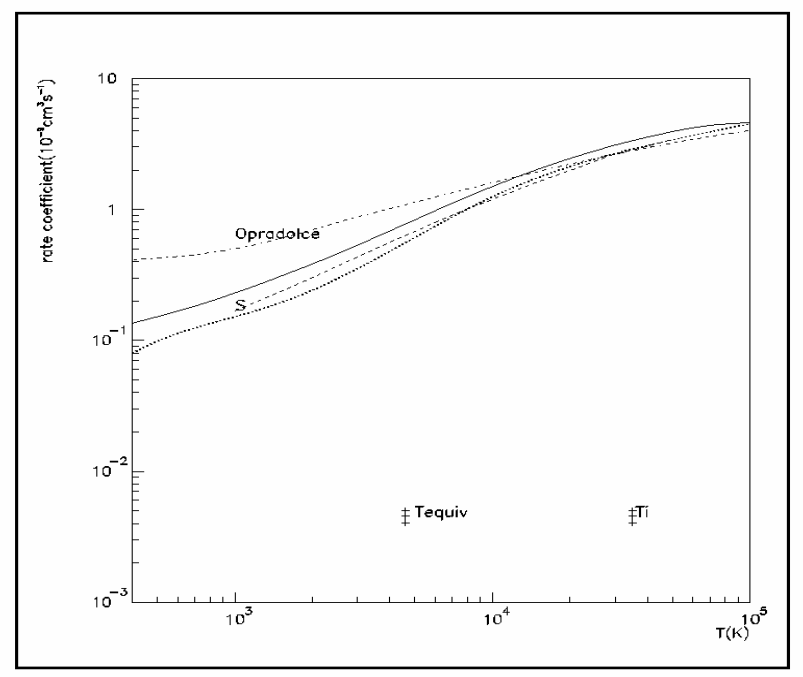

Fig. 8 : Rate coefficients as a function of temperature for $\mathrm{Si}^{4+}+\mathrm{He}$. Comparison of ion trap experiment at $\mathrm{T}_{\mathrm{i}}$ and $\mathrm{T}_{\text {equiv }}$ [15] with calculations using time-dependent (full line : Hankel-Ricatti functions [6] ; dotted line : plane wave functions [5]) or time-independent methods (S : Stancil et al. [26] ; Opradolce et al. [29]). 
by the $T_{i}$ and $T_{\text {equiv }}$ temperatures presented on the graph, but this could never explain such a discrepancy. As shown for the $\mathrm{O}^{2+}+\mathrm{He}$ system, the existence of an excited state could eventually explain a two order difference in rate coefficients, but the first excited state, $\mathrm{Si}^{3+}\left(2 \mathrm{p}^{5} 3 \mathrm{~s}\right)$, is definitely too high in energy to be a reasonable candidate. In conclusion, it appears clearly that all the theoretical approaches converge to the same result but cannot explain the experimental data.

\section{Conclusions}

This work presents a comparative study between recent theoretical approaches and ion-trap experiments which provide for the first time a direct determination of rate coefficients at low temperature. For $\mathrm{N}^{2+}+\mathrm{He}$, a good agreement is found between ion-trap experiments and ab-initio calculations, but for three of the collisional systems considered here $\left(\mathrm{O}^{2+}, \mathrm{Si}^{3+}\right.$ and $\mathrm{Si}^{4+}$ on He target $)$, the theoretical values show significant differences with ion trap experimental data, from $40 \%$ for $\mathrm{Si}^{3+}$, to one or two orders of magnitude for $\mathrm{O}^{2+}$ and $\mathrm{Si}^{4+}$. The difficulty to determine precisely the temperature of the reactants in the trap appears to be a fundamental difficulty in such experiments, associated with the assumption of a nearly thermal ion velocity distribution. A more appropriate description of the energy distribution in the ion trap must be considered. The eventual presence of excited states or impurity in the trap could also be suggested with respect to the production of the ions, but it seems unlikely considering the long waiting time before collecting data. However, despite all these possible sources of imprecision, the very large discrepancies between the experimental data of Fang and Kwong and all the theoretical predictions in the case of the $\mathrm{Si}^{4+}+\mathrm{He}$ collisional system is still totally unaccounted for.

\section{References}

1. Honvault, P.; Bacchus-Montabonel, M.C.; Gargaud, M.; McCarroll, R. Chem. Phys. 1998, 238, 401.

2. Stancil, P.C.; Clarke, N.J.; Zygelman, B.; Cooper, D.L. J. Phys. B : At. Mol. Opt. Phys. 1999, 32, 1523.

3. Gargaud, M.; Bacchus-Montabonel, M.C.; McCarroll, R. J. Chem. Phys. 1993, 99, 4495.

4. Vaeck, N.; Desouter-Lecomte, M.; Liévin, J. J. Phys. B : At. Mol. Opt. Phys. 1999, 32, 409.

5. Vaeck, N.; Bacchus-Montabonel, M.C.; Baloïtcha, E.; Desouter-Lecomte, M. Phys. Rev. 2001, A63, 042704.

6. Baloïtcha, E.; Desouter-Lecomte, M.; Bacchus-Montabonel, M.C.; Vaeck, N. J. Chem. Phys. 2001, 114, 8741 .

7. Havener, C.C.; Huq, M.S.; Krause, H.F.; Schultz, P.A.; Phaneuf, R.A. Phys. Rev. 1989, A39,1725.

8. Olamba, K.; Szücs, S.; Chenu, J.P.; El Arbi, N.; Brouillard, F. J. Phys. B : At. Mol. Opt. Phys. 1996, 29, 2837.

9. Kwong, V.H.S.; Fang, Z. Phys. Rev. Lett. 1993, 71, 4127.

10. Fang, Z.; Kwong, V.H.S. Phys. Rev. 1995, A51, 1321.

11. Gao, H.; Fang, Z.; Kwong, V.H.S. Phys. Rev. 2001, A63, 032704.

12. Fang, Z.; Kwong, V.H.S. Astrophys. J. 1997, 474, 529. 
13. Fang, Z.; Kwong, V.H.S. Phys. Rev. 1997, A55, 440.

14. Fang, Z.; Kwong, V.H.S. Astrophys. J. 1997, 483, 527

15. Fang, Z.; Kwong, V.H.S. Phys. Rev. 1999, A59, 342

16. Fang, Z.; Chen, D.; Kwong, V.H.S. Phys. Rev. 2000, A62, 042709.

17. Kwong, V.H.S.; Fang, Z. Astrophys. J. 2001, 536, 954.

18. Kimura, M.; Gu, J.P.; Hirsch, G.; Buenker, R.J. Phys. Rev. 1996, A53, 4164.

19. Butler, S.E.; Heil, T.G.; Dalgarno, A. J. Chem. Phys. 1984, 80, 4986.

20. Wang, J.G.; Stancil, P.C.; Kingdon, J.B.; Rakovic', M.; Schultz, D.R.; Dalgarno, A. 2002 (to be submitted).

21. Sun, Y.; Sadeghpour, H.R.; Kirby, K.; Dalgarno, A. Inter. Rev. Phys. Chem. 1996, 15, 53.

22. Lafyatis, G.P.; Kirby, K.; Dalgarno, A. Phys. Rev. 1993, A48, 321.

23. Bacchus-Montabonel, M.C. Theor. Chem. Acc. 2000, 104, 296.

24. Butler, S.E.; Dalgarno, A. Astrophys. J. 1980, 241, 838.

25. Bacchus-Montabonel, M.C.; Ceyzeriat, P. Phys. Rev. 1998, A58, 1162.

26. Stancil, P.C.; Zygelman, B.; Clarke, N.J.; Cooper, D.L. Phys. Rev. 1997, A55, 1064.

27. Suzuki, R.; Watanabe, A.; Sato, H.; GU, J.G.; Hirsch, G.; Buenker, R.J.; Kimura, M.; Stancil, P.C. Phys. Rev. 2001, A63, 042717.

28. Tawara, H.; Okuno, K.; Fehrenbach, C.W.; Verzani, C.; Stockli, M.P.; Depaola, B.D.; Richard, P.; Stancil, P.C. Phys. Rev. 2001, A63, 062701.

29. Opradolce, L.; McCarroll, R.; Valiron, P. Astron. Astrophys. 1982, 106, 197.

30. Parlant, G.; Yarkony, D.R. Int. J. Quantum Chem. 1992, Symp.26, 737.

31. Lengsfield III, B.H.; Yarkony, D.R. Adv. Chem. Phys. 1991, 82, 1.

32. Mandelshtam, V.A.; Taylor, H.S. J. Chem. Phys. 1995, 102, 7390; Mandelshtam, V.A. J. Phys. Chem, 2001, 105, 2764.

33. Tal-Ezer, H.; Kosloff, R. J. Chem. Phys. 1984, 81, 3967.

34. Feit, M.D.; Fleck, J.A.; Steiger, A. J. Comput. Phys. 1982, 47, 412.

35. Jäckle A.; Meyer, H.D. J. Chem. Phys. 1996, 105, 6778.

36. Burns, D.; Thompson, W.R.; Greenwood, J.B.; McCullough, R.W.; Geddes, J.; Gilbody, H.B. J. Phys. B : At. Mol. Opt. Phys. 1997, 30, 4559.

37. Lee, A.R.; Wilkins, A.C.R.; Enos, C.S.; Brenton, A.G. Phys. Rev. 1993, A48, 2934.

38. Sadilek, M.; Vancura, J.; Farnik, J.; Herman, Z. Int. J. Mass Spectrom. Ion Processes 1990, 100, 197.

39. McLaughlin, T.K.; Wilson, S.M.; McCullough, R.W.; Gilbody, H.B. J. Phys. B : At. Mol. Opt. Phys. 1990, 23, 737.

40. Bacchus-Montabonel, M.C.; Courbin, C.; McCarroll, R. J. Phys. B : At. Mol. Opt. Phys. 1991, 24, 4409.

41. Johnsen, R.; Biondi, M.A. J. Chem. Phys. 1981, 74, 305.

42. McLean, A.D.; Chandler, G.S. J. Chem. Phys. 1980, 72, 5639.

43. Pelissier, M.; Komiha, N.; Daudey, J.P. J. Comput. Chem. 1988, 9, 298.

44. Clarke, N.J.; Cooper, D.L. J. Chem. Soc. Faraday Trans. 1998, 94, 3295.

45. MOLPRO (version 98.1) is a package of ab-initio programs written by Werner, H.J. and Knowles, P. with contributions from Almlöf, J.; Amos, R.D.; Deegan, M.J.O.; Elbert, S.T.; Hampel, C.; Meyer, W.; Peterson, K.; Pitzer, R.; Stone, A.J.; Taylor, P.R.; Lindh, R.. 
46. Dunning, T.H. J. Chem. Phys. 1989, 90, 1007.

47. Hay, P.J.; Wadt, W.R. J. Chem. Phys. 1985, 82, 270

(C) 2002 by MDPI (http://www.mdpi.org), Basel, Switzerland. 